\section{Efecto del fotocurado con luz led en la filtración marginal de un sellante de fosas y fisuras}

\author{
Effect of LED curing on the marginal leakage of a pit and fissure sealant
}

Artículo OriginAL

\section{Martha Elena Pineda Mejía ${ }^{1,2}$, Waldo Gloria Zevallos², Tulio Abuhadba Hoyos ${ }^{2}$}

1 Magister en Estomatología. Profesor Principal. UNMSM.

2 Dpto. Estomatología Rehabilitadora. Facultad Odontología. UNMSM.

\section{Correspondencia:}

Facultad Odontología. Ciudad Universitaria UNMSM. Lima 1 - Perú.

Correo electrónico: mpineda@unmsm.edu.pe ésta diferencia no mostró significancia estadística $(p>0.05)$. El fotocurado con luz L.E.D. o con luz halogena convencional tienen efectos similares sobre el grado de filtracion marginal de un sellante de fosas y fisuras

Palabras clave: Sellantes, diodos, filtración.

\begin{abstract}
The purpose of this study was to evaluate in vitro the curing effect of a light-emitting diode LED unit and conventional halogen light on the marginal leakage of a pit and fissure sealant. We used 40 caries-free permanent premolars recently extracted, which were randomly divided into two groups $(\mathrm{n}=20)$, sealed with a resin based fissure sealant, Helioseal F (Ivoclar Vivadent), according to the manufacturer patterns, then photopolymerized using either L.E.D. light or conventional halogen light (control group). The specimens were stored in distilled water at $37^{\circ} \mathrm{C}$ for 48 hours, and covered all tooth surfaces with nail enamel except for a margin of $2 \mathrm{~mm}$ around the sealants, the samples were then immersed in a $1 \%$ blue methylene dye solution as a marginal leakage indicator, and subjected to thermocycling regimen of 500 cycles between $5{ }^{\circ} \mathrm{C}$ and $60{ }^{\circ} \mathrm{C}$. After removal of the dye solution specimens were sectioned lingual to vestibule way, to evaluated marginal leakage on a stereomicroscope. $35 \%$ and $60 \%$ of the sealants photopolymerized using either L.E.D. light or conventional halogen light showed some degree of marginal leakage, but the difference was not statistically significant ( $\mathrm{p}>.05)$. The curing L.E.D. light or conventional halogen light has similar effects on the marginal leakage degree of a pit and fissure sealant.
\end{abstract}

Keywords: Sealants, diodes, filtration.

\section{Introducción}

Se sabe que la alta susceptibilidad de los dientes a la caries, puede ser atribuida entre otros factores a la complicada morfología de las fosas y fisuras, las cuales, son consideradas como el sitio ideal para la retención de bacterias y de residuos alimenticios, además por su ubicación resultan ser inaccesibles cuando se realiza la remoción mecánica ${ }^{1,2}$

De acuerdo a su morfología, las fosas y fisuras dentales constituyen verdaderos nichos para que los microorganismos se alojen en ellas, y sean el punto de partida en la aparición y desarrollo de la ca- ries dental. La placa dental sólo puede ser removida por encima de la entrada de la superficie de la fisura, siempre y cuando se realice una muy buena técnica de cepillado dental. Las regiones profundas de las fisuras, son áreas de retención para la placa y cuando esta no es removida con el cepillado, las lesiones cariosas pueden aparecer y desarrollarse ${ }^{2,3}$. Por tanto, se ha considerado importante conocer cómo la morfología dental de las fosas y fisuras influyen en el desarrollo de la caries dental, y la necesidad de realizar algún tipo de tratamiento de prevención para evitar su aparición.
Fecha de recepción: 11-06-2013

Fecha de aceptación: 04-09-2013
El cierre de las fosas y las fisuras de las superficies dentarias por medio de sustancias adhesivas como son los sellantes de fosas y fisuras, constituye un procedimiento preventivo y terapéutico de extraordinario valor, estos están formulados a base de resina con carga inorgánica, que luego permanecen firmemente unidos al esmalte, aunque una de las objeciones es la duda sobre la capacidad de retención del sellador ${ }^{8}$.

Los sellantes tienen 3 efectos preventivos fundamentales: ${ }^{8}$

1) Obturan mecánicamente las fosas $y$ fisuras con una resina resistente a los ácidos 
2) Al obturar las fosas y fisuras suprimen el hábitat de los streptococcus mutans y otros microorganismos.

3) Facilita la limpieza de las fosas y fisuras mediante métodos físicos como el cepillado dental y la masticación.

El principal factor a tener en cuenta para la aplicación de un sellante es el diagnóstico del estado de salud de las fosas y fisuras que se pretenden cerrar, lo cual a menudo se vuelve un reto, especialmente con los ultimos cambios en el enfoque del diagnóstico y tratamiento de la caries. En los últimos ańos, el concepto de repasar un explorador afilado por las fosas y fisuras esta siendo descartado en favor de la observacion visual del esmalte, el diagnóstico radiográfico, y el uso de nuevos instrumentos de diagnóstico. Incluso con las nuevas tecnologías para el diagnóstico de caries, aun es difícil determinar la progresión de la enfermedad, ya que cuando las caries de fosas y fisuras se examinan histologicamente, se observa una variacion considerable. ${ }^{16}$

Para iniciar la reacción química de polimerización de estos materiales se requiere de un aporte de energía a través de algún mecanismo de iniciación que puede ser activación química (autocurado), activación por calor (termocurado) y activación por luz visible (fotocurado) ${ }^{4}$

Para este último caso, existen varias fuentes lumínicas: ${ }^{4}$

- Lámparas de plasma (de arco, xenón).

- Lámparas de láser.

- Lámparas halógenas: Las que pueden ser convencionales (hasta 400$500 \mathrm{~mW} / \mathrm{cm}^{2}$ ) y de alta intensidad o potencia (sobre $500 \mathrm{~mW} / \mathrm{cm}^{2}$ ).

- Lámparas de luz emitida por diodos (L.E.D): Con una potencia que va desde los 800 a los $1500 \mathrm{~mW} / \mathrm{cm}^{2}$.

La luz para el curado de materiales dentales estéticos más común hoy en día es la luz halógena de tungsteno de cuarzo $(\mathrm{QTH})^{6}$. El relativo amplio espectro de las lámparas halógenas, les permite iniciar la polimerización de todos los materiales dentales de resina disponibles en la actualidad. La principal salida de estas lámparas es la energía infrarroja con la generación de alto calor. Los filtros se utilizan para reducir la disipación de calor a través de las estructuras orales y para proporcionar una restricción adicional de la luz visible al estrecho espectro de fotoiniciadores. Con altas temperaturas de funcionamiento, los bombillos QTH tienen una vida útil limitada, sin embargo continúan proporcionando un método fiable $y$ rentable de fotopolimerización. A pesar de la popularidad, la tecnología de la bombilla halógena, tiene algunos inconvenientes. Las lámparas halógenas tienen una vida efectiva limitada de alrededor de 40-100 horas. La bombilla, el reflector y el filtro se degradan con el tiempo debido a la alta temperatura desarrollada, lo que lleva a una reducción de la salida de luz. Esto se traduce en una reducción de la efectividad de la luz de la unidad de curado. ${ }^{7,8}$

Ultimamente las lámparas de tipo L.E.D. se están incorporando como una alternativa más eficiente según sus fabricantes. Este nuevo sistema de fuente lumínica aparece en el mercado odontológico alrededor del ańo 1995. Son lámparas que emiten una luz azul con una longitud de onda entre 440$490 \mathrm{~nm}$, con un peak de $460 \mathrm{~nm}^{3}$, permitiendo activar un fotoiniciador que sea sensible a esa longitud de onda y producir la polimerización del material. ${ }^{5}$

En lugar del filamento caliente usado en las bombillas halógenas, LED utiliza uniones de semiconductores diodos para la generación de la luz y tienen una vida útil de más de 10.000 horas y sufren poca degradación de salida durante ese tiempo. No requieren de filtros para producir la luz azul, son resistentes a los golpes y vibraciones, consumen poca energía en su funcionamiento y son inalámbricos 9,10,11. La luz de curado LED utiliza semiconductores especiales para la electroluminiscencia de la luz en lugar de un filamento caliente como se hace en las unidades halógenas QTH. ${ }^{12}$ Esto proporciona una vida más larga, consistencia de salida y menor consumo de potencia ${ }^{13}$, el cual permite tener unidades de peso ligero para la mano, y ser alimentadas por batería. Ninguna luz ultravioleta o infrarroja significante es emitida, reduciendo así el calor lateral, minimizando la necesidad de un ruidoso ventilador. ${ }^{14}$

El elemento fotoiniciador de la reacción de polimerización más comúnmente utilizado en las resinas compuestas es la Canforoquinona. Ésta absorbe energía a un espectro de luz visible entre $400 \mathrm{y}$ $500 \mathrm{~nm}$., con un peak ideal de $468 \mathrm{~nm}$, valor que coincide con el espectro emitido por las lámparas L.E.D., lo que les daría a éstas una mayor eficiencia en la fotoactivación ${ }^{4}$. Por lo tanto, la probabilidad de que un fotón emitido por una lámpara L.E.D. sea absorbido por las canforoquinonas es considerablemente mayor a que si fuera de una lámpara halógena convencional. Esto eventualmente se traduciría en que la lámpara L.E.D. lograría producir una fotoactivación de mayor eficiencia y, por lo tanto, una polimerización más completa. ${ }^{4}$

La potencia de las lámparas L.E.D. oscila entre los 1000-1400 $\mathrm{mW} / \mathrm{cm} 2$, a pesar de que sólo se necesita 300-400 $\mathrm{mW} / \mathrm{cm} 2$ para lograr una buena polimerización. ${ }^{6}$ Debido a su alta potencia los fabricantes aseguran que, en comparación con una lámpara halógena convencional, las lámparas L.E.D. logran una mayor polimerización de las resinas compuestas con un menor tiempo de exposición lumínica ${ }^{7}$. Sin embargo, esto podría traducirse en una activación muy brusca, generando tensiones que pueden afectar la calidad del sellado marginal de la restauración.

Las lámparas halógenas de uso convencional emiten luz a su máxima potencia desde el momento en que se encienden, lo que obliga a maniobras de compensación. Las lámparas LED, por lo contrario, poseen la cualidad de ir incrementando su potencia en forma automática en los primeros 5 segundos de exposición, con lo que el material restaurador lograría una mejor adaptación marginal, que podría reflejarse en un mejor sellado marginal.

El propósito de éste estudio es evaluar in vitro el efecto del fotocurado con luz L.E.D. y luz halogena convencional sobre la filtracion marginal de un sellante de fosas y fisuras.

\section{Material y métodos}

Se seleccionaron 40 premolares sin caries recientemente extraídas por razones ortodoncicas, las cuales después de pasar por un proceso de limpieza se mantuvieron en una solución de hipoclorito de sodio desde el momento de la exodoncia hasta el momento de iniciar la experimentación.

Los dientes fueron aleatoriamente distribuidos en dos grupos:

G1: $(n=20)$ se les colocó sellante de fisura fotoactivado con luz L.E.D.

G2: $(n=20)$ se les colocó sellante de fisura fotoactivado con luz halogena.

Este procedimiento fue realizado por un solo operador entrenado y calibrado.

El sellante de fosas y fisuras utilizado fue HELIOSEAL' F (IVOCLAR VI- 
VADENT, LIECHTENSTEIN) que se compone de una matriz de monomero de BIS-GMA, dimetacrilato de uretano y trietilenglicoldimetacrilato $(58.6 \%$ en peso). El material de rellleno se compone de dioxido de silicio altamente disperso y vidrio de fluorosilicato (40.5\% en peso). Ademas contiene dioxido de titanio, estabilizadores y catalizadores (<1\% en peso).

\section{Fotoactivacion:}

Se utilizó una lámpara de luz emitida por diodos L.E.D. GUILIN WOODPECKER MEDICAL INSTRUMENT CO. Ltd. P.R. Guilin, Guangxi, China, con una potencia de $650 \mathrm{~mW} / \mathrm{cm}^{2}$, y una lámpara de luz halógena convencional marca Litex 680A (Dentamerica Industry, CA 91744 USA), con una potencia de $480 \mathrm{~mW} / \mathrm{cm}^{2}$.

Protocolo de la colocación del sellante :

- Se empleo una tecnica no invasiva .

- Lavado y secado preliminar de la pieza dentaria.

- Profilaxia del esmalte de la corona

- Grabado ácido: con ácido ortofosfórico al $37 \%$ en gel, el cual fue aplicado con una jeringa en las fosas y fisuras por $30 \mathrm{seg}$.

- Lavado, secado con aire sin grasa ni agua.

- Aplicación directa del sellante en las fosas y fisuras con la canula de la jeringa dispensadora y polimerizado con lámpara de fotoactivación según grupo experimental, por 40 segundos.

Filtración y termociclado:

Una vez colocados los sellantes, los dientes se dejaron en la estufa a $37^{\circ}$ Celsius, al $100 \%$ de humedad durante 48 horas, luego se cubrieron todas las superficies de la pieza dentaria con esmalte para uńas excepto un margen de $2 \mathrm{~mm}$ alrededor de los sellantes. Luego se hizo un proceso de termociclado de las muestras, utilizando una solución acuosa de azul de metileno al $1 \%$ usado como indicador de la microfiltración marginal, el régimen fue de 500 ciclos entre $5^{\circ}$ y $60^{\circ} \mathrm{C}$ manteniendo las muestras 30 segundos en cada bańo térmico.

Después de este proceso se procedió al lavado y secado de las muestras para posteriormente ser cortadas longitudinalmente en sentido vestivulo palatino, con el fin de analizar la filtración marginal en la interfase diente sellante.
Evaluacion de la filtración marginal: se hizo en un microscopio estereoscopio Leitz Carl Zeiss, con un aumento de (40X). La calificacion de los grados de filtración de tinta (Fig.1.) se hizo mediante los siguientes criterios:

$$
\begin{aligned}
& 0=\text { no hay filtración } \\
& 1=\text { filtración a la mitad de la fisura } \\
& 2=\text { filtración total }
\end{aligned}
$$

\section{Resultados}

Los resultados de la filtración se muestran en la tabla 1 y gráfico 1 . La gran mayoría de sellantes fotocurados con luz LED no mostraron filtración marginal (grado 0). En ambos grupos se encontró grado 3 de filtración. El $35 \%$ y $60 \%$ de los sellantes fotoactivados con luz LED y luz halógena respectivamente presentaron algún grado de micro filtración marginal, sin embargo, no hubo diferencia estadísticamente significante ( $>0.05)$ para las pruebas $\mathrm{X}^{2}$ y KruskalWallis a un nivel de confianza del $95 \%$. aparentemente sanas (caries ocultas) sean equivocadamente sellados ${ }^{13}$. El sellado marginal es importante para el éxito del sellador porque la penetración de las bacterias bajo el sellador podría permitir la aparición de caries y/o la progresión de ésta ${ }^{14,15}$.

En este estudio, se evaluó la microfiltración in vitro de un sellante de fosas y fisuras a base de resina HELIOSEAL F, fotocurado con luz LED y con luz halógena. Todos los materiales se aplicaron sin ameloplastía con el fin de observar el comportamiento de estos materiales sin la eliminación de la sustancia dental. Los especímenes fueron termociclados para reproducir las diferentes temperaturas a las que los dientes están sometidos durante el comer y beber bajo condiciones clínicas.

En el $65 \%$ y $40 \%$ de especímenes del grupo LED y luz halógena respectivamente, no se produjo microfiltración marginal del sellante, ésta amplia diferencia nos hace pensar que la profundidad de penetración de la luz LED fue

Tabla 1. Distribución de los grupos según grados de filtración marginal

\begin{tabular}{ccccc}
\hline Grado & 0 & 1 & 2 & Total \\
\hline L.E.D & $1365 \%$ & $630 \%$ & $105 \%$ & $20100 \%$ \\
Halogena & $840 \%$ & $945 \%$ & $315 \%$ & $20100 \%$ \\
\hline
\end{tabular}
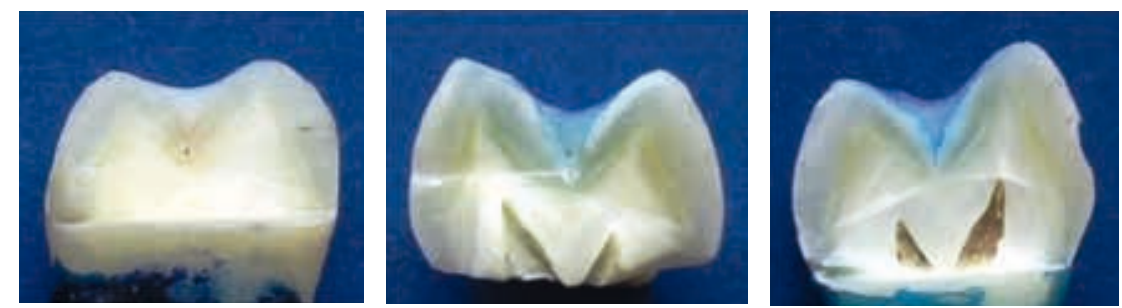

Fig. 1. Grados de filtración marginal cero, uno y dos respectivamente.

\section{Discusión}

Los sellantes de fosas y fisuras son considerados un complemento excelente para las estrategias preventivas de salud bucal para disminuir el inicio o progreso de la caries oclusal ${ }^{4}$. Las propiedades ideales de un material de sellado incluyen biocompatibilidad, la retención, la resistencia a la abrasión y el desgaste ${ }^{8}$, para muchos, el uso de un agente de unión al esmalte es también importante porque la microfiltración en la interfase diente-material puede conducir al fracaso del tratamiento.

De acuerdo con la Asociación Dental Americana $^{12}$, los sellantes de fisuras pueden ser colocados en caries incipientes de esmalte. Sin embargo, es posible que los dientes con lesiones de la dentina bajo superficies de esmalte mayor. Además, las lámparas halógenas de uso convencional emiten luz a su máxima potencia desde el momento en que se encienden. Las lámparas LED, por lo contrario, poseen la cualidad de ir incrementando su potencia en forma automática en los primeros 5 segundos de exposición, con lo que el material colocado estaria logrando una mejor adaptación marginal, que se refleja en un mejor sellado marginal.

Los grados de filtración marginal 1 y 2 se presentaron en el $35 \%$ del grupo luz LED y en el $60 \%$ del grupo halógena, si bien en este último grupo la tendencia fue a presentar mayor cantidad de especímenes filtrados, las diferencias no mostraron significancia estadística para las pruebas chi cuadrado y KruskallWallis ( $p>0.05)$. La polimerización con las lámparas LED puede ser más lenta 
que con las lámparas halógenas, pero los resultados pueden ser similares o mejores y la comodidad en la manipulación de las primeras es superior.

Comportamientos similares obtuvieron, Nalcaci A; Ulusoy N; Kücükesmen $\mathrm{C}^{4}$ realizaron un estudio in vitro para evaluar el efecto de fotocurado de una unidad de luz emitida por diodos LED de muy alta intensidad, con otra LED de intensidad convencional y una unidad de luz halogena convencional, empleando y diferentes modos de exposicion, en el polimerizado del sellante de fisura Fissurit-F. Para lo cual emplearon molares permanentes libres de caries, divididos en seis grupos experimentales. Los resultados no mostraron diferencia estadísticamente significante en la microfiltracion marginal de un sellante de fosas y fisuras en la comparacion de las diferentes tecnicas de fotocurado empleadas, tampoco que los regimenes de termociclado hayan tenido algun efecto en ya sea especimenes curados con las diferentes intensidades de luz LED o halogena convencional.

Cehreli SB; Arikan S; Gulsahi K; Arhun N; Arman A; Sargon M. ${ }^{6}$ realizaron una investigacion in vitro con el proposito de evaluar la microfiltracion marginal de un sellante de fisuras a base de ormocer (Admira Seal) con dos diferentes unidades de luz emitida por diodos LED y una unidad de luz halogena quarzo-tungsteno convencional, para lo cual emplearon 30 terceras molares permanentes sanas de adolescentes, conformando tres grupos experimentales, en uno se polimerizó el sellante con luz LED (Smarth Light) por 10 segundos, en otro grupo se hizo el polimerizado con luz LED (Elipar II) por 10 segundos y en el tercero con luz halogena (Hilux) por 30 segundos. Los resultados no mostraron diferencias estadisticamente significantes entre los grupos de experimentacion. Las unidades de luz LED pueden proporcionar una reducción en el tiempo de aplicación total sin comprometer la integridad del sellado marginal del sellante de fisuras a base de ormocer.

Salama FS; Al-Hmmad NS ${ }^{7}$, realizaron un estudio in vitro con el propósito de evaluar el sellado marginal y el grado de penetración dentro de la fisura, de un sellante a base de resina (Concise) y un compomero restaurativo (F2000) con y sin ameloplastia, para lo cual em- plearon 30 terceras molares permanentes sanas, conformando seis grupos de experimentación. Los resultados mostraron que la microfiltracion es similar cuando el sellante se coloca con grabado acido y el compomero con grabado acido mas agente de union, con o sin ameloplastia. La penetracion del sellante es mejor cuando se usa ameloplastia y grabado acido

En definitiva, el rendimiento de las lámparas LED es muy similar al de las halógenas, pero puede lograr una polimerización de mayor profundidad. Además, el poco aumento de temperatura implica menor dańo pulpar durante el tratamiento, lo cual es un dato a tener muy en cuenta y de aplicabilidad inmediata en el atención clínica de pacientes odontológicos.

\section{Conclusiones}

Bajo las condiciones del presente estudio in vitro, el fotocurado con luz L.E.D. o con luz halogena convencional tienen efectos similares sobre el grado de filtracion marginal de un sellante de fosas y fisuras

\section{Referencias bibliográficas}

1. Urquia MM, Brasca N, Girardi M; Bonnin C, Rios M. In vitro study of microleakage of fissure sealant with different previous treatments. Acta OdontolLatinoam 2011; 24 (2): 150-4.

2. Markovic D, Petrovic B, Peric T, Miletic I, Andjelkovic S. The impact of fissure depth and enamel conditioning protocols on glassionomer and resin-based fissure sealant penetration. J Adhes Dent. 2011; 13(2): 171-8.

3. Kane B, Karren J, Garcia-Godoy F. Sealant adaptation and penetration into occlusal fissures. Am J Dent 2009; 22(2): 89-91.

4. Nalcaci A, Ulusoy N, Kusukesmen C. Effect of LED curing modes on the microlekage of a pit and fissure sealant. Am J Dent 2007; 20(4): 255-8.

5. Kersten S, Lutz F, Schupbach P. Fissure sealing: optimization of sealant penetration and sealing properties. Am J Dent 2001; (3): 127-31.

6. Cehreli SB, Ghulsahi K, Arthun N, Arman A, Sargon M. Effect of LED curing on marginal integrity of on ormocer-based sealant. J Dent Child Chic 2009; 76(1): 537.

7. Salama FS, Al-Hammad NS. Marginal seal of sealant and compomer materials with and without enameloplasty. Int J Paediatr 2002; 12(1): 39-46.

8. Barrancos MJ. Operatoria Dental. 3era ed. Ed. Médica Panamericana; 1999. 454-470 p.

9. Macchi RL. Materiales Dentales. 3era ed. Ed. Médica Panamericana; 2000. 117-123 p.

10. Schwartz RS, Summitt JB, Robbins JW. Fundamentos en Odontología Operatoria. Un logro contemporáneo. Ed. Actualidades Médico Odontológicas Latinoamérica; $1999.56 \mathrm{p}$.

11. Uribe Echeverri J. Operatoria Dental. Ciencia y práctica. Ed. Avances Médico-Dentales; 1990. 71-89 p.

12. American Dental Association. Dental sealants. ADA Council on access, prevention and interprofessional relations; ADA council on scientific affairs. J Am Dent Assoc 1997; 128: 485-488 p.

13. Weerheijm KL, De Soet JJ, De Graaff J, Van Amerongen WE. Occlusal hidden caries: a bacteriological profile. J Dent Child 1990; 57(6): 428-32.

14. Jensen OE, Handelman SL. Effect of an autopolymerizing sealant on viability of microflora in occlusal dental caries. Scand J Dent Res 1980; 88(5): 382-8.

15. Weerheijm KL, Groen HJ. The residual caries dilemma. Community Dent Oral Epidemiol 1999; 27(6): 436-41.

16. Strassler HE, Grebosky M, Porter J, Arroyo J. Success with pit and fissure sealants. Dentistry Today; 31 de enero de 2005 [Citado 17 de julio de 2012]. Disponible en: http://www.dentistrytoday.com/ ce-articles/351 